\title{
Contribution
}

\section{Quantitative Detection of Cerebral Arteriosclerosis by the Ultrasonic Doppler Technique: Criteria for the Diagnosis of Cerebral Arteriosclerosis}

\author{
Manabu MIYAZAKI \\ Department of Internal Medicine Miyazaki Naika Clinic
}

\begin{abstract}
The correlation between continuity of cerebral blood flow velocity (CBFV) pattern (internal carotid artery) and cerebral vascular resistance, indicating cerebral arteriosclerosis $(\mathrm{CA})$, was investigated by the ultrasonic Doppler technique. A significant correlation between continuity of the CBFV pattern and the severity of CA was recognized: the greater the decrease in continuity of the CBFV pattern, the greater the severity of CA. In a previous study, the continuous index (CI) of the CBFV pattern was devised as an objective parameter of the continuity of the CBFV pattern for evaluating cerebral hemodynamics in CA. Pulsatility index (PI) and resistance index (RI) are similar in nature to the CI. These parameters are useful as objective indexes of cerebral vascular resistance, indicating CA, particularly of the arteriole. Among these parameters, PI seems to be more useful clinically than CI and RI. Criteria for the quantitative detection of CA, particularly of the arteriole, are proposed by calculation of the PI.
\end{abstract}

Key words: continuous index $(\mathrm{Cl})$, pulsatility index $(\mathrm{PI})$, resistance index (RI)

\section{Introduction}

Quantitative detection of cerebral arteriosclerosis (CA) will play an import role in the treatment of cerebral vascular diseases. However, no suitable technique has yet been developed in this field because of methodological difficulties.

Examination of the cerebral blood flow velocity (CBFV) pattern by the ultrasonic Doppler technique is the easiest method for the quantitative detection of CA. This method is based on a significant correlation between continuity of the CBFV pattern and the severity of $\mathrm{CA}^{2-7)}$.

In a previous study in 1967 , the continuous index (CI) of the CBFV pattern was devised as an objective parameter of continuity of the CBFV pattern (cerebral vascular resistance), and CA was examined using this parameter ${ }^{2-7)}$. In further studies, Gosling et al. ${ }^{1)}$ and Taylor et al. ${ }^{13)}$ described the pulsatility index (PI) and resistance index (RI), which are similar in nature to the CI.

In this study, the quantitative detection of CA was examined using the ultrasonic Doppler technique, with a view to developing criteria for the quantitative detection of $\mathrm{CA}$, particularly of the arteriole.

\section{Correlation between continuous index, pulsatility index and resistance index}

Correlation between $C I$ and PI

CI, PI and RI were calculated by the methods shown in Fig. 1 (A, B, C).

In the equations presented in Fig. 1, when $\mathrm{X}$ and $\mathrm{Y}$ are set down as constant, $\mathrm{X} / \mathrm{Y}=\mathrm{A}$ and $\mathrm{Y}-\mathrm{X}=\mathrm{B}, \mathrm{CI}$ and $\mathrm{PI}$ are calculated as $\mathrm{CI}=\mathrm{A} \times \mathrm{Z}$ and $\mathrm{PI}=\mathrm{B} /$ mean, respectively. Generally speaking, mean $\propto \mathrm{Z}$, so PI $=\mathrm{B} /$ mean $\propto \mathrm{B} / \mathrm{Z}=\mathrm{A} \times \mathrm{B} / \mathrm{CI}$. Therefore, $\mathrm{PI} \propto 1 / \mathrm{CI}$. In conclusion, it is suggested that an inverse correlation exists between $\mathrm{CI}$ and PI.

Indeed, a significant inverse correlation was recognized between CI and PI in clinical practice using 30 young healthy volunteers as well as elderly patients with cerebral vascular diseases $(r=-0.803, p<0.01, Y=2.427-0.035 X)(\text { Fig. } 2)^{9}{ }^{9}$.

\section{Correlation between $C I$ and $R I$}

In the equations presented in Fig. 1, when $\mathrm{X}$ and $\mathrm{Y}$ are set down as constant, $\mathrm{X} / \mathrm{Y}=\mathrm{A}$ and $\mathrm{Y}-\mathrm{X}=\mathrm{B}, \mathrm{CI}$ and $\mathrm{RI}$ are calculated as $\mathrm{CI}=\mathrm{A} \times \mathrm{Z}$ and $\mathrm{RI}=1-\mathrm{A}=1-\mathrm{CI} / \mathrm{Z}$, respectively. Under conditions where no significant changes are recognized in pulse rate, $\mathrm{Z}$ can be regarded as constant, and RI $\propto$-CI. This indicates that a negative correlation exists between $\mathrm{CI}$ and RI. 
(A) Continuous index $(\mathrm{Cl})$ Continuous Index $(\mathrm{Cl})$

$$
=\frac{X Z}{Y}
$$

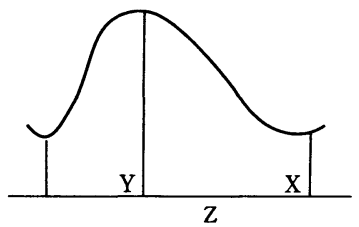

$X:$ smallest amplitude in diastole

Y: largest amplitude in systole

$Z$ : duration of pulse rate
(B) Pulsatility index $(\mathrm{PI})$ Pusatility Index (PI)

$$
=\frac{Y-X}{\text { Mean }}
$$

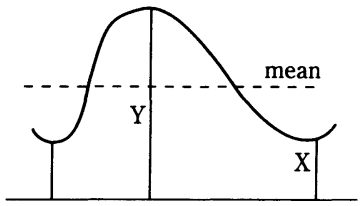

$X$ : smallest amplitude in diastole

$Y$ : largest amplitude in systole

Mean: mean amplitude in the pulse cycle
(C) Resistance index (RI) Resistance Index (RI)

$$
=\frac{\mathrm{Y}-\mathrm{X}}{\mathrm{Y}}
$$

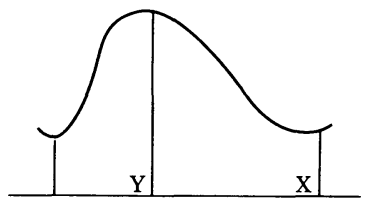

$X:$ smallest amplitude in diastole

$Y$ : largest amplitude in systole

Fig. 1 Calculation of continuous index (CI), pulsatility index (PI) and resistance index (RI).

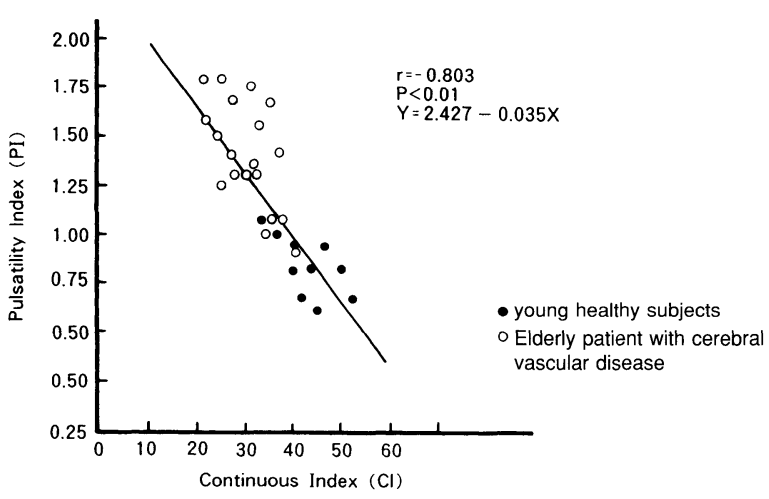

Fig. 2 Correlation of continuous index (CI) and pulsatility index (PI) of cerebral blood flow pattern (internal carotid artery) in young healthy subjects and elderly patients with cerebral vascular diseases.

The above results support that idea that CI, PI and RI are similar in nature and that these parameters may be useful as objective parameters of cerebral vascular resistance suggesting CA.

\section{Problems with CI, PI and RI}

When these parameters are applied clinically, the calibration of heart rate is most important. $\mathrm{Z}$, the interval of one cardiac cycle in CI, and mean, the mean blood flow velocity of one cardiac cycle in PI, are important factors in the calibration of heart rate.

On the other hand, no calibration of heart rate in RI is a demerit of RI. RI seems to give rise to non-negligible errors in severe arrhythmia such as tachycardia and bradycardia.

As for CI, the following considerations are essential: (i) CI cannot be calibrated at zero point of $\mathrm{X}$ (blood flow in diastole is arrested); and (ii) PI and RI change in parallel with cerebral vascular resistance. On the other hand, CI changes inversely with change in cerebral vascular resistance.

In conclusion, $\mathrm{PI}$ is considered more suitable than $\mathrm{CI}$ and $\mathrm{RI}$ as an objective parameter of cerebral vascular resistance
A

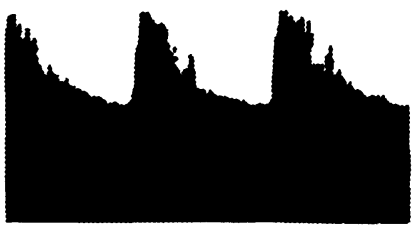

B
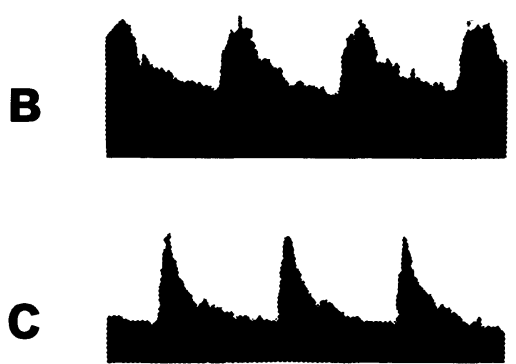

Fig. 3 Classification of cerebral blood flow velocity pattern (internal carotid artery)

(A) Continuous type, (B) intermediate type, (C) discontinuous type.

suggesting $\mathrm{CA}$.

\section{Correlation of CBFV pattern (internal carotid artery) and cerebral vascular resistance}

\section{Clinical investigation}

The CBFV patterns (internal carotid artery) measured by the Doppler ultrasonic technique were classified according to continuity of the CBFV pattern into the following three types: (i) continuous type; (ii) intermediate type; and (iii) discontinuous type (Fig. 3).

Correlation between continuity of the CBFV pattern and the severity of CA was investigated by comparing intracerebral arteriosclerosis with extracerebral arteriosclerosis anatomically, and with retinal arteriosclerosis, clinically. The results showed a significant correlation between continuity of the CBFV pattern and the severity of $\mathrm{CA}$ : the greater the decrease in continuity of the CBFV pattern, the greater the severity of $\mathrm{CA}^{2,7,9-12)}$.

Table 1 and Fig. 3 show criteria for the quantitative detection of $\mathrm{CA}$ by the ultrasonic Doppler method (continuity method). 
Table 1. Criteria for the quantitative detection of cerebral arteriosclerosis by the ultrasonic Doppler technique

\begin{tabular}{|c|c|c|}
\hline $\begin{array}{l}\text { Type of cerebral blood } \\
\text { flow velocity pattern }\end{array}$ & Severity of cerebral arteriosclerosis & Note \\
\hline Continuous type & $\begin{array}{c}\text { Extracerebral atherosclerosis and } \\
\text { intracerebral arteriosclerosis are } \\
\text { absent or minimal }\end{array}$ & $\begin{array}{c}\text { Pseudo- } \\
\text { continuous type }\end{array}$ \\
\hline Intermediate type & $\begin{array}{l}\text { Extracerebral atherosclerosis and } \\
\text { intracerebral arteriosclerosis may } \\
\text { belong to the continuous and } \\
\text { discontinuous types }\end{array}$ & $\begin{array}{l}\text { Pseudo- } \\
\text { intermediate } \\
\text { type }\end{array}$ \\
\hline Discontinuous type & $\begin{array}{l}\text { Extracerebral atherosclerosis and } \\
\text { intracerebral arteriosclerosis are } \\
\text { severe. Among them, intracerebral } \\
\text { arteriosclerosis is more severe } \\
\text { than extracerebral atherosclerosis }\end{array}$ & $\begin{array}{l}\text { Pseudo- } \\
\text { discontinuous } \\
\text { type }\end{array}$ \\
\hline
\end{tabular}

Table 2. Criteria for the quantitative detection of cerebral arteriosclerosis by pulsatility index (PI) measurement (internal carotid artery)

\begin{tabular}{|c|c|c|}
\hline Age & PI & Cerebral arteriosclerosis \\
\hline & $<1.3$ & Normal artery \\
$<60$ years & $1.3-1.7$ & Mild arteriosclerosis \\
& $1.7-2.0$ & Moderate arteriosclerosis \\
& $>2.0$ & Severe arteriosclerosis \\
\hline & $<1.4$ & Normal artery \\
$>60$ years & $1.4-1.8$ & Mild arteriosclerosis \\
& $1.8-2.1$ & Moderate arteriosclerosis \\
& $>2.1$ & Severe arteriosclerosis \\
\hline
\end{tabular}

Continuous index (CI) of the CBFV pattern was devised as an objective parameter of continuity (i.e., cerebral vascular resistance and $\mathrm{CA}$ ).

\section{Basic investigation}

Alterations of blood flow velocity patterns were investigated in the following circulatory stress tests: (i) hand-grasping; (ii) brachial-binding; and (iii) cold and warm-stimulating tests. It was revealed that the more peripheral vascular resistance was increased, the more discontinuity of the brachial blood flow velocity pattern increased.

Fig. 4 shows the alterations of CI and PI in the brachial artery examined by the antebrachial binding test ("manchette" method). A significant decrease of CI and increase of PI in the brachial artery were observed in response to an increase in antebrachial binding using a cuff. Alterations of CI and PI in the blood flow velocity pattern in the brachial artery were also observed in the other examinations (hand-grasping, cold and warm-stimulating test).

It is postulated from the above clinical and basic investigations that continuity of the CBFV pattern and the three parameters, CI, PI and RI, seem to be useful as objective parameters of cerebral vascular resistance (i.e., CA, particular of the arteriole).

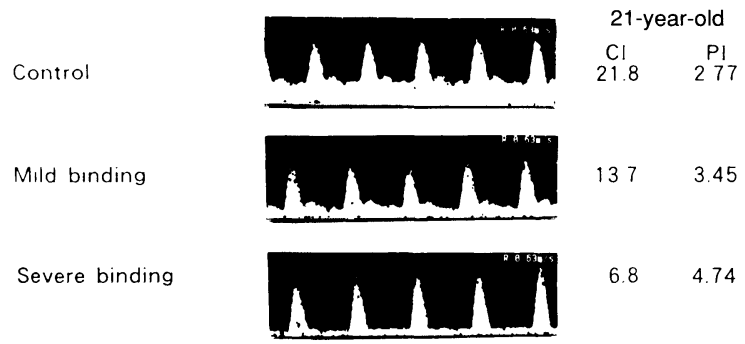

Fig. 4 Change of continuous index (CI) and pulsatility index (PI) in the antebrachial-binding test (brachial artery).

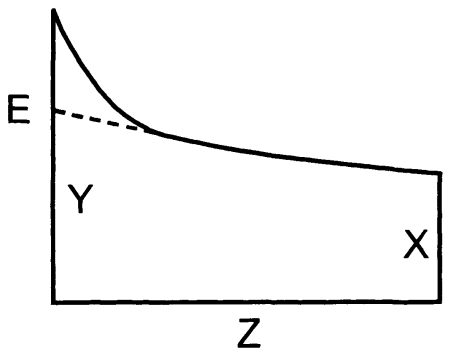

Fig. 5 E-Point (exponential point) is detected instead of Y-point, in case the initial spike is observed in the cerebral blood flow velocity pattern.

\section{Criteria for quantitative detection of CA by measurement of PI}

The correlation between PI of the CBFV pattern measured by the ultrasonic Doppler technique and risk factors of cerebral vascular diseases (e.g., aging, hypertension, diabetes mellitus and hypercholesterolemia) was investigated and a significant correlation was recognized between PI and the risk factors.

Table 2 shows criteria for the quantitative detection of $\mathrm{CA}$, particularly of the arteriole, by the PI measurement technique. When applying these criteria, the differences in PI between the vessels (internal carotid artery, external carotid artery, common carotid artery, internal jugular vein, etc.), architecture and variation of the vessels should be considered.

In general, PI in the internal carotid artery is smaller than that in the external and common carotid arteries. On the other hand, PI in the internal jugular vein seems to be smaller than PI in the internal carotid artery.

An initial large spike may occasionally be observed at the onset of the blood flow velocity pattern (Fig. 5). In this case, it may be appropriate to detect the E-point (exponential point) instead of the usual Y-point. As for the mechanisms responsible for this spike, several factors such as cardiac function and elasticity of the carotid artery may be involved. 


\section{Conclusion}

The three parameters, CI, PI and RI, of the CBFV pattern measured by the ultrasonic Doppler technique are similar in nature. These parameters are useful as objective parameters of cerebral vascular resistance suggesting $\mathrm{CA}$, particular of the arteriole.

PI seems to be more suitable than CI and RI from the standpoint of clinical usefulness. Criteria for the quantitative detection of CA, particularly of the arteriole, by measurement of the PI are proposed.

\section{References}

1) Gosling RG, King DH, Newman DL, Woodcock JP: Transcutaneous measurement of arterial blood velocity by ultrasound. In Ultrasonics for Industry Conference Papers 1969, pp16-23

2) Miyazaki M: Cerebral circulatory disorders and correlation to several organs from the viewpoint of Doppler ultrasonic blood flow measurement. Igaku-Journal Co., 1981

3) Miyazaki M: Quantitative detection of cerebral arteriosclerosis by the Doppler ultrasonic technique. Naika 19: 733-738, 1967

4) Miyazaki M: Quantitative detection of cerebral arteriosclerosis by the Doppler ultrasonic technique. Jpn. Circulation J. 31: 781-786, 1967

5) Miyazaki M: Quantitative detection of cerebral arteriosclerosis by means of the Doppler ultrasonic technique. Gerontology 32 (suppl. I): 17-23, 1986.
6) Miyazaki M: Quantitative detection of cerebral arteriosclerosis by means of the Doppler ultrasonic technique. Basic and clinical investigation in man. Japan Medical Journal 349: 31-34, 1991

7) Miyazaki M: Measurement of cerebral vascular resistance and its clinical application by means of the Doppler ultrasonic technique. Japan Medical Journal. 3569: 24-27, 1992

8) Miyazaki M: Quantitative detection of cerebral arteriosclerosis by means of the Doppler ultrasonic technique. Recent advances in neurosonology. Oka. M(ed), Elsevier, 1992, pp373-377

9) Miyazaki M: Quantitative detection of cerebral arteriosclerosis by the Doppler ultrasonic technique. Japan Medical Journal 3130: 27-31, 1984

10) Miyazaki M: Quantitative detection of cerebral arteriosclerosis by means of the Doppler ultrasonic technique. Correlation of CI and PI. Japan Medical Journal. 3454: 15-18, 1990

11) Miyazaki M: Effect of cerebral vasodilator on cerebral circulation. Japan Medical Journal. 3517: 29-31, 1991

12) Miyazaki M: Detection of cerebral circulatory homeostasis by means of the Doppler ultrasonic technique with special reference to carotid compression test. Japan Medical Journal. 3555: 22-24, 1992

13) Taylor KJW Burns PN: Duplex Doppler scanning in the pelvis and abdomen. Ultrasound in Medicine and Biology 11: 643-658, 1985 